\title{
Chemoradiotherapy and Increased Prescription Dose in Esophageal Squamous Cell Cancer: A Retrospective Study
}

\author{
Xiaofen Pan, ${ }^{1}$ Mingchu Liao, ${ }^{2}$ Hongmei Ma, ${ }^{3}$ Xiaobing Jiang, ${ }^{4}$ Hanwen Huang, ${ }^{5}$ Min Wei $\mathbb{D}^{1}$, \\ and Qun Li $\mathbb{1}^{6}$ \\ ${ }^{1}$ Department of Oncology, The Seventh Affiliated Hospital, Sun Yat-sen University, Shenzhen, China \\ ${ }^{2}$ Department of Medical Oncology, The First Affiliated Hospital of University of South China, Hengyang, China \\ ${ }^{3}$ Department of Medical Oncology, The First People's Hospital of Chengdu, Chengdu, China \\ ${ }^{4}$ Department of Neurosurgery/Neuro-oncology, Sun Yat-sen University Cancer Center, State Key Laboratory of Oncology in \\ South China, Collaborative Innovation Center for Cancer Medicine, Guangzhou, China \\ ${ }^{5}$ Department of Oncology, Yunfu People's Hospital of Guangdong, Yunfu, China \\ ${ }^{6}$ Department of Radiation Oncology, Sun Yat-sen University Cancer Center, State Key Laboratory of Oncology in South China, \\ Collaborative Innovation Center for Cancer Medicine, Guangzhou, China
}

Correspondence should be addressed to Min Wei; 171319146@qq.com and Qun Li; liqun0799@163.com

Received 30 June 2020; Revised 21 December 2020; Accepted 24 January 2021; Published 8 February 2021

Academic Editor: Kosei Maemura

Copyright (c) 2021 Xiaofen Pan et al. This is an open access article distributed under the Creative Commons Attribution License, which permits unrestricted use, distribution, and reproduction in any medium, provided the original work is properly cited.

\begin{abstract}
To analyze the outcomes and adverse events of patients with esophageal squamous cell carcinoma (ESCC) treated with definitive chemoradiation with modified radiotherapy volume and increased radiation dose. This was a retrospective analysis of patients with ESCC treated with definitive chemoradiotherapy at the Sun Yat-sen University Cancer Center (02/2015 to 02/2017). The dose to the planning gross tumor volume (PGTV) and planning clinical tumor volume (PTV1) was 66-68 Gy (2.0-2.2 Gy/fraction). The dose to the planning regional lymph node drainage area volume (PTV2) was $46 \mathrm{~Gy}$ (2.0 Gy/fraction). Treatment response, adverse events, progression-free survival (PFS), overall survival (OS), and locoregional failure-free survival (LRFFS) were analyzed. Twenty-six patients were included. The median follow-up was 31 (range, 4.3-51.3) months. Sixteen (61.5\%) patients had a complete response, and four (15.4\%) achieved a partial response. The objective response rate was $76.9 \%$, and the disease control rate was 80.8\%. The median PFS and OS were not achieved. The 4-year PFS was 63.9\%, and the 4-year OS was 71.0\%. Grade 1-2 and 3-4 radiation-related esophagitis was observed in 15 (57.7\%) and one (4.5\%) patients, respectively. Grade 1-2 and 3-4 radiationrelated pneumonitis was observed in $12(46.2 \%)$ and one (4.5\%) patients, respectively. No patients developed radiation-related heart or skin damage. The modified target volume definition and increased dose of definitive radiotherapy combined with chemotherapy in patients with ESCC had low toxicity and might improve survival, but additional trials are necessary to prove the superiority of this strategy.
\end{abstract}

\section{Introduction}

Esophageal cancer is the eighth leading cause of cancer death in the world [1]. Esophageal squamous cell carcinoma (ESCC) and adenocarcinoma are the two common subtypes of esophageal cancer. In East Asia, ESCC is the most common type of esophageal cancer, while adenocarcinoma is more prevalent in western countries [2]. These two types differ in pathogenesis, prognosis, and tumor biology [3].
For early-stage esophageal cancer, surgery remains the standard therapy, but the patients still have 3-5-year survival rates of 5\%-32\% [4]. The combination of chemotherapy and radiotherapy improves local control and overall survival (OS) rates in medically fit populations [5]. In Europe and North America, 50.4 Gy is the standard dose for a patient undergoing definitive chemoradiation, based on the RTOG 90-12 and INT0123 trials [6]. Even though dose escalation showed no improvement to local control and OS, $60.0 \mathrm{~Gy}$ remains a commonly used dose in Asian countries [7], where ESCC is 
more common than in western countries where most trials were conducted [2]. A pooled analysis showed that the median progression-free survival (PFS) ranged from 7.4 to 12.0 months, and OS ranged from 9.0 to 34.0 months; the 1 -, 2-, and 3 -year pooled OS rates were $57 \%, 39 \%$, and $40 \%$, respectively [8].

Intensity-modulated radiation therapy (IMRT) has the advantage of limiting the radiation dose to organs at risk without reducing the dose delivered to the tumor [9]. IMRT has a benefit in decreasing pulmonary complications, but IMRT failed to show a cancer-specific survival over threedimensional radiation (3DCRT) when using the current standard radiation therapy doses [10]. A study from the MD Anderson Cancer Center showed the failure patterns in patients treated by IMRT based on modern-day radiation treatment volumes [9]. The study indicated that $50 \%$ of patients had a local failure, while $48 \%$ had distant failures. Among the local failure cases, 90\% were within the gross tumor volume (GTV), and 23\% were within the clinical target volume (CTV) [9].

Therefore, the present study is aimed at analyzing the outcomes and adverse events of patients with ESCC treated with definitive chemoradiation with radiotherapy volume using modified GTV and CTV definitions and increased radiation dose.

\section{Materials and Methods}

2.1. Study Design and Patients. This was a retrospective analysis of patients with ESCC treated with definitive chemoradiotherapy at the Sun Yat-sen University Cancer Center between February 2015 and February 2017. This study was approved by the Ethics Committee of Sun Yat-sen University Cancer Center.

The inclusion criteria were as follows: (1) newly diagnosed and histologically proven ESCC [11], (2) $\geq 18$ years of age at start therapy, (3) tumor was unsuitable for esophagectomy [12], (4) definitive chemoradiotherapy using IMRT, and (5) the target volume delineating method was modified according to the description in the radiation therapy part. The exclusion criteria were as follows: (1) history of other tumors, (2) treated for recurrent disease, (3) had distant metastasis (not including celiac or supraclavicular lymph nodes), or (4) received other $2 \mathrm{D}$ or $3 \mathrm{D}$ radiation therapy.

2.2. Radiation Therapy. The GTV includes the primary tumor (GTVt) and involved regional lymph nodes (GTVnd). The GTVt encompassed the primary tumor according to the CT scan and endoscopy. The volume of lymph nodes (GTVnd) encompassed lymph nodes in the drainage area shown by the CT scan, disregarding the diameters.

The CTV1 was defined as $3 \mathrm{~cm}$ above the proximal edge and $3 \mathrm{~cm}$ below the distal edge of the GTVt and 1-2 $\mathrm{mm}$ from the GTVt, outside the esophageal wall. The upper border of the CTV did not expand above the cricoid cartilage, and the lower border did not expand to the stomach unless a gross tumor was present at those levels. CTV2 for regional lymph nodal drainage area volumes was contoured according to the expert consensus contouring guidelines [13] (Figure 1).

The planning gross target volume (PGTVt) was defined as $0.5 \mathrm{~cm}$ from the GTVt in the superior-inferior directions and $0.5 \mathrm{~cm}$ in the right-left and the anteroposterior directions. The planning gross tumor volume of lymph nodes (PGTVnd) was defined as $0.5 \mathrm{~cm}$ from the GTVnd in the superior-inferior, right-left, and anteroposterior directions. The planning target volumes of CTV1 (PTV1) and CTV2 (PTV2) were defined as $0.5 \mathrm{~cm}$ from the CTV1 and CTV2 in the superior-inferior, right-left, and anteroposterior directions. The modified definitions of target volumes are shown in Table 1.

Radiation therapy was administered by IMRT technology with a total dose of 66-68 Gy delivered to the PGTVt and PGTVnd. The radiation therapy plan was designed as a one-stage or two-stage strategy. For the one-stage strategy, a dose of 44-46 Gy was delivered to the PTV2, and a dose of 66-68 Gy was delivered to the PGTVt, PGTVnd, and PTV1 by a simultaneously boost technology. For the twostage strategy, a dose of $46 \mathrm{~Gy}$ was delivered to the PTV1, PTV2, PGTVt, and PGTVnd in conventional fractionation of $2.0 \mathrm{~Gy} /$ fraction in the first stage (stage A), and a dose of 20-22 Gy was delivered to the PGTVt, PGTVnd, and PTV1 in a fraction of 2.0-2.2 Gy/fraction in the second stage (stage B) right after stage $A$.

The lung V20 was defined as the proportion of lung volume receiving irradiation of $\geq 20$ Gy to the total lung volume. In general, a one-stage design was first performed, and, then, the lung V20 was evaluated. If the lung V20 exceeded 30\%, a two-stage design was performed to control the lung V20 to be within 30\%. Later, it was found that the two-stage design of the patient's lung V20 was lower than the one-stage design, so the two-stage design was performed for patients later on. But regardless of the one- or two-stage design, the total radiation dose was the same.

Radiation therapies were performed by a radiation therapist with more than 30 years of experience in this field.

2.3. Chemotherapy. Some patients were scheduled for induction chemotherapy or concurrent chemotherapy. Chemotherapy consisted of paclitaxel/nedaplatin, paclitaxel/5fluorouracil/nedaplatin, docetaxel/5-fluorouracil/nedaplatin, vinorelbine+cisplatin, pemetrexed/nedaplatin, vinorelbine/S-1, or S-1 monotherapy, as per the NCCN guidelines [12].

2.4. Follow-Up. The first follow-up visit was 2-3 months after treatment. Additional follow-up was offered every 3-4 months for the first two years and every 6-12 months thereafter. Follow-up was ended in August 2019.

2.5. Observational Outcomes. For basic information, sex, age, tumor location, $\mathrm{T}$ and $\mathrm{N}$ stages, and clinical stage were recorded. TNM stages were all restaged according to the $8^{\text {th }}$ edition of the AJCC TNM classification. For treatmentrelated characteristics, chemotherapy regimens, combined with Endostar or not, numbers of induction chemotherapy cycles, and radiation doses were recorded. 

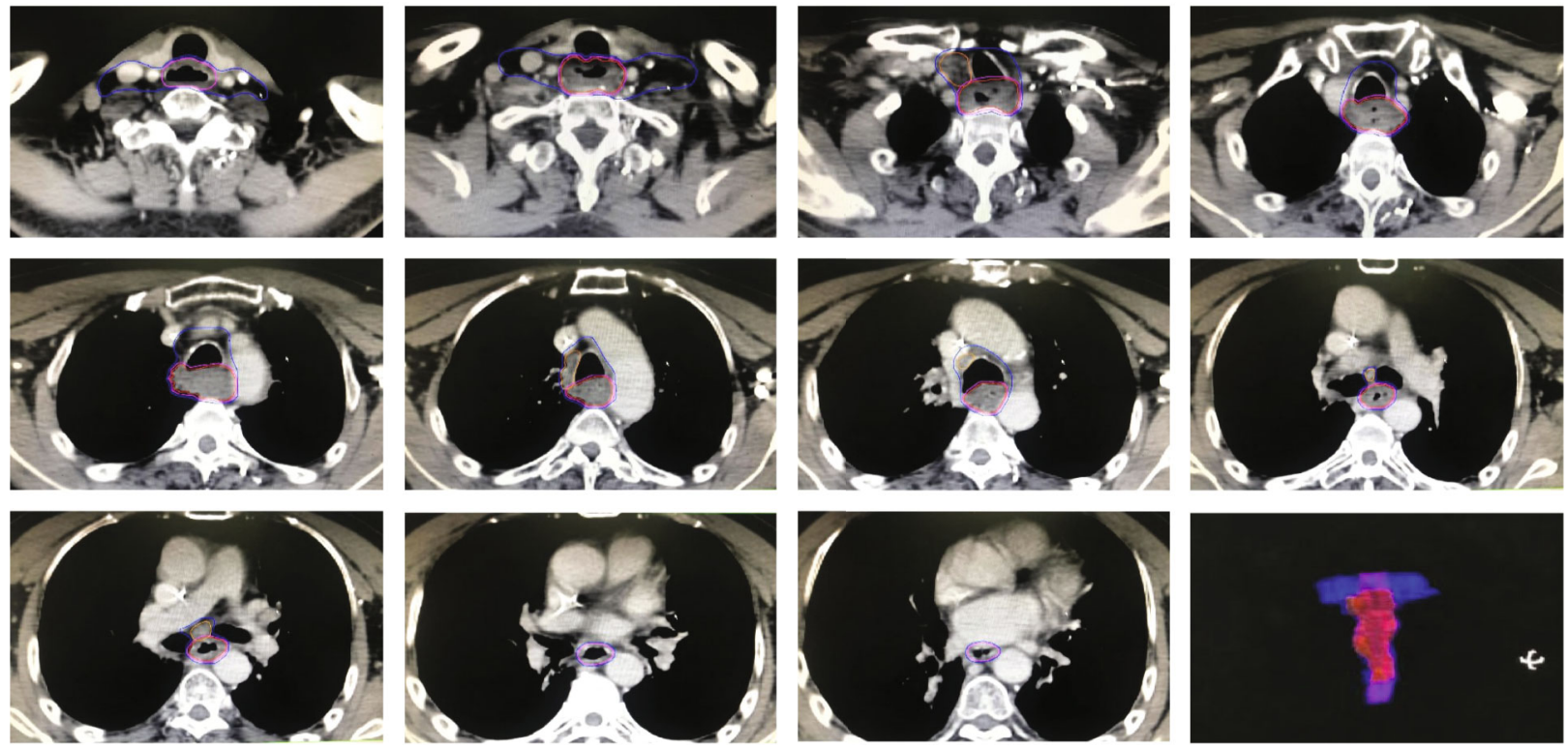

Figure 1: Definition of target volumes. Primary tumor gross tumor volume (GTVt) is shown as the red line. The involved regional lymph node gross tumor volume (GTVnd) is shown as the orange line. The clinical tumor volume (CTV1) is shown as a pink line. The clinical tumor volume for regional nodal volume (CTV2) is shown as the blue line. The planning gross target volume (PGTVt), planning gross tumor volume of lymph nodes (PGTVnd), planning clinical tumor volume (PTV1), and planning clinical tumor volume for regional nodal volume (PTV2) were a 0.5-centimeter expansion from GTVt, GTVnd, CTV1, and CTV2 (PTVs were not shown).

TABle 1: Target volume definitions.

\begin{tabular}{|c|c|c|c|}
\hline & NCCN guideline definition ${ }^{\#}$ & Modified definition & Prescription dose \\
\hline GTV & $\begin{array}{c}\text { Primary tumor (GTVt) and involved lymph } \\
\text { node (GTVnd) }\end{array}$ & $\begin{array}{c}\text { Primary tumor (GTVt) and involved lymph } \\
\text { node (GTVnd) }\end{array}$ & $66-68 \mathrm{~Gy}, 2.0-2.2 \mathrm{~Gy} /$ fraction \\
\hline CTV & $\begin{array}{l}3-4 \mathrm{~cm} \text { expansion above and below the edge of } \\
\text { GTVt and } 1 \mathrm{~cm} \text { expansion around the } \\
\text { esophageal wall as primary tumor CTV. The } \\
\text { nodal GTV plus an expansion of } 0.5-1.5 \mathrm{~cm} \text { as } \\
\text { the nodal CTV. Elective nodal regions should } \\
\text { be included. }\end{array}$ & $\begin{array}{l}3 \mathrm{~cm} \text { expansion above and below the edge of } \\
\text { the GTVt and } 1-2 \mathrm{~mm} \text { expansion around the } \\
\text { esophageal wall as CTV1. Elective nodal } \\
\text { regions defined as CTV } 2 \text { and GTVnd should } \\
\text { include in CTV2. }\end{array}$ & $\begin{array}{c}\text { CTV1: 66-68 Gy, } 2.0- \\
\text { 2.2 Gy/fraction;CTV2: } 46 \text { Gy, } \\
\text { 2.0 Gy/fraction }\end{array}$ \\
\hline
\end{tabular}

PTV 0.5-1 cm expansion
"Esophageal and esophagogastric junction cancers, V2.2019.

Tumor response and acute adverse events after treatment were followed and recorded. Complete response (CR), partial response (PR), stable disease (SD), and progressive disease (PD) were evaluated by CT scan and endoscopy, according to RECIST 1.1 [14]. CR: all target lesions disappeared. PR: the total diameter of all target lesions is reduced by $\geq 30 \%$. PD: the total diameter of all target lesions is increased by $\geq 20 \%$. SD: all target lesions did not reach $P R$, and the increase did not reach $\mathrm{PD}$. Objective response rate $(\mathrm{ORR})=$ $\mathrm{CR}+\mathrm{PR}$. Disease control rate $(\mathrm{DCR})=\mathrm{CR}+\mathrm{PR}+\mathrm{SD}$.

Adverse events mainly included hematologic toxicity, heart damage, esophagitis, pneumonitis, and skin damage and were graded according to the National Cancer Institute Common Terminology Criteria for Adverse Events (NCI CTCAE) version 3.0 [15].

Locoregional failure was defined as a relapse/progression of the primary tumor or regional lymph nodes. Distant failure was defined as tumor metastasis to distant sites. Any occurrence of this condition during follow-up was considered a failure.

OS and PFS were recorded. OS was defined as the time from treatment initiation to death or loss to follow-up or end of follow-up. PFS was defined as the time from treatment initiation to disease progression (including locoregional failure and distant failure), loss to follow-up, or end of followup. Locoregional failure-free survival (LRFFS) was defined as the time from treatment initiation to locoregional failure, loss to follow-up, or end of follow-up.

2.6. Statistical Analysis. SPSS 19.0 (IBM, Armonk, NY, USA) was used for statistical analysis. Continuous variables are presented as medians (range). Categorical variables are reported as frequencies (\%). OS, PFS, and LRFFS were shown according to the Kaplan-Meier method, and subgroup analyses were conducted using the log-rank test. $P$ values $<0.05$ were considered statistically significant. 


\section{Results}

3.1. Characteristics of the Patients. Twenty-six patients were included and analyzed. The median age was 58.5 (range, 39-81) years, $21(80.8 \%)$ patients were male, and five (19.2\%) were female. Twenty-three $(88.5 \%)$ patients were scheduled for induction chemotherapy, and all patients received at least one cycle of concurrent chemotherapy. The characteristics of the patients are shown in Table 2.

3.2. Treatment Effect. Median follow-up was 31 (range, 4.351.3) months for all patients and 40.4 (range, 30.6-51.3) months for survivors. All patients completed radiotherapy without any interruption longer than 7 days. Treatment characteristics are shown in Table 3. Sixteen (61.5\%) patients had $\mathrm{CR}$, four (15.4\%) achieved PR, one (3.8\%) had SD, two (7.7\%) developed PD, and three (11.5\%) were not evaluable due to missing data. The ORR was $76.9 \%$, and the DCR was $80.8 \%$ (Table 4 ).

3.3. Survival. The median PFS and OS were not reached when the present analyses were performed. The 1-, 2-, and 3-year PFS rates were $91.3 \%, 68.5 \%$, and $63.9 \%$, respectively. The 1 -, 2-, and 3-year OS rates were 92.3\%, 75.4\%, and 71.0\%, respectively. The 1-, 2-, and 3-year LRFFS rates were $96.2 \%$, $73.3 \%$, and $73.3 \%$, respectively. The 4 -year PFS was $63.9 \%$, the 4 -year LRFFS rate was $73.3 \%$, and the 4 -year OS was $71.0 \%$, indicating stable survival from 3 to 4 years in those patients (Figures 2(a)-2(c)). The patients with recurrence underwent systemic treatment based on their supervising physicians; they did not undergo further irradiation therapy.

Subgroup analyses were performed, and the results are shown in Table 5 and Figures 2(d)-2(f). The results show that male sex was associated with a poorer 3-year PFS $(P=0.034$ vs. female sex $)$, middle-thoracic tumor localization was associated with a poorer 3 -year OS $(P<0.001$ vs. the other localizations), and clinical stage III was associated with a poorer 3 -year OS $(P=0.010$ vs. stage IV).

3.4. Failure Pattern Analysis. Six (23.1\%) patients experienced locoregional failure. Five (19.2\%) patients had only locoregional failure, and one $(3.8 \%)$ had both locoregional and distant failure. Four patients failed at the primary site, and two patients failed in the regional lymph nodes. Three patients developed distant metastases: bone $(n=2)$ and liver $(n=1)$.

3.5. Adverse Events. Toxicities related to treatments are summarized in Table 6. No patients developed radiation-related heart or skin damage. Sixteen $(61.5 \%)$ patients developed grade 1-2 hematologic toxicity, and seven (26.9\%) developed grade 3-4 hematologic toxicity. Grade 1-2 radiation-related esophagitis was observed in $15(57.7 \%)$ patients, and grade 3-4 radiation-related esophagitis was observed in one (4.5\%) patient. Grade 1-2 radiation-related pneumonitis was observed in $12(46.2 \%)$ patients, and grade 3-4 radiation-related pneumonitis was observed in one $(4.5 \%)$ patient. No patients died of radiation-related toxicities.
TABLE 2: Characteristics of the patients.

\begin{tabular}{lcc}
\hline Characteristics, $n(\%)$ & & Patients $(n=26)$ \\
\hline \multirow{2}{*}{ Sex } & Male & $21(80.8)$ \\
& Female & $5(19.2)$ \\
\hline \multirow{2}{*}{ Age (years) } & $\geq 75$ & $3(11.5)$ \\
& $<75$ & $23(88.5)$ \\
\hline \multirow{4}{*}{ Tumor localization } & Cervical & $4(15.4)$ \\
& Upper thoracic & $8(30.8)$ \\
& Middle thoracic & $11(42.3)$ \\
& Lower thoracic & $3(11.5)$ \\
\hline \multirow{3}{*}{ T stage } & 1 & 0 \\
& 2 & $2(8.7)$ \\
& 3 & $8(30.8)$ \\
& 4 & $13(50.0)$ \\
N stage & $\mathrm{x}$ & $3(11.5)$ \\
\hline \multirow{3}{*}{ Clinical stage } & N0 & 0 \\
& N+ & $26(100)$ \\
\hline
\end{tabular}

\section{Discussion}

According to an expert consensus [13] and NCCN guidelines [12], the GTV should include the primary tumor and involved regional lymph nodes based on CT images, clinical information, and PET-CT images. CTV should be $3-4 \mathrm{~cm}$ above and $3-4 \mathrm{~cm}$ below the edge of the GTV. The nodal CTV should expand by $0.5-1 \mathrm{~cm}$ from the nodal GTV. For distal esophageal or gastroesophageal junction cancer, in order to reduce the radiation volume to the stomach and other organs, a margin of $2 \mathrm{~cm}$ below the GTV edge is acceptable. The radial border should be $1 \mathrm{~cm}$ from the outer wall of the esophagus. If the GTV covers essential organs such as the heart and liver, the CTV expansion should be limited to $0.5 \mathrm{~cm}$. For regional nodal volumes, the CTV should be contoured according to the location of the tumor. For distal tumors approaching or involving the gastroesophageal junction, the CTV should cover the celiac, para-aortic, and gastrohepatic lymph nodes. For tumors above the carina, bilateral supraclavicular nodal areas should be included in the CTV, and the recommended borders are to level IV lymph nodes in head and neck cancer [16]. The lower paratracheal nodal stations and upper paratracheal nodal stations and prevascular nodal stations, which correspond to IASLC levels 2, 4, and 3, respectively [17], should also be included. Base on the NCCN guideline, the recommended dose of definitive radiation is 50-50.4 Gy $(1.8-2.0 \mathrm{~Gy} / \mathrm{d})$ [12].

Nevertheless, when using those treatment parameters, the prognosis of unresectable esophageal cancer is still poor. The 5-year survival is about $25 \%$, and the local recurrence rate is about $40-60 \%$ after definitive CRT $[5,6,18]$. The 1- 
TABLE 3: Characteristics of the treatments.

\begin{tabular}{|c|c|c|}
\hline Characteristics & & Patients $(n=26)$ \\
\hline \multirow{2}{*}{ Chemotherapy regimens, $n(\%)$} & $\mathrm{TP}^{\#}$ & $20(76.9)$ \\
\hline & Others* & $6(23.1)$ \\
\hline \multirow{2}{*}{ Combine with Endostar, $n(\%)$} & Yes & $14(53.8)$ \\
\hline & No & $12(46.2)$ \\
\hline \multirow{4}{*}{ Number of induction chemotherapy cycles, $n(\%)$} & 0 & $3(11.5)$ \\
\hline & 1 & $4(15.4)$ \\
\hline & 2 & $16(61.5)$ \\
\hline & $\geq 3$ & $3(11.5)$ \\
\hline \multirow{2}{*}{ Radiation therapy, $n(\%)$} & One-stage strategy & $13(50)$ \\
\hline & Two-stage strategy & $13(50)$ \\
\hline \multirow{3}{*}{ Radiation dose (cGy) } & Median (range) & $6800(6620-6930)$ \\
\hline & $>6800, n(\%)$ & $7(26.9)$ \\
\hline & $\leq 6800, n(\%)$ & $19(73.1)$ \\
\hline
\end{tabular}

${ }^{\#}$ TP, paclitaxel+nedaplatin. ${ }^{*}$ Other chemotherapy regimens included TPF (paclitaxel+nedaplatin+S-1, $\left.n=1\right)$; DPF (docetaxel+nedaplatin+S-1, $\left.n=1\right) ; \mathrm{NP}$ (vinorelbine+cisplatin, $n=1$ ); PP (pemetrexed+nedaplatin, $n=1$ ); vinorelbine+S-1, $n=1$; S-1 monotherapy, $n=1$.

TABLE 4: Tumor response assessment*.

\begin{tabular}{lccccc}
\hline $\begin{array}{l}\text { Clinical } \\
\text { stage }\end{array}$ & $\begin{array}{c}\text { All } \\
(n=26)\end{array}$ & $\begin{array}{c}\text { IIB } \\
(n=2)\end{array}$ & $\begin{array}{c}\text { III } \\
(n=15)\end{array}$ & $\begin{array}{c}\text { IV } \\
(n=8)\end{array}$ & $\begin{array}{c}\mathrm{X} \\
(n=1)\end{array}$ \\
\hline CR & 16 & 1 & 8 & 7 & 0 \\
PR & 4 & 0 & 3 & 1 & 0 \\
SD & 1 & 0 & 1 & 0 & 0 \\
PD & 2 & 0 & 1 & 0 & 1 \\
Not & 3 & 1 & 2 & 0 & 0 \\
evaluable & 76.9 & 50 & 73.3 & 100 & 0 \\
ORR (\%) & 80.8 & 50 & 80.0 & 100 & 0 \\
DCR (\%) & & & & & 0 \\
\hline
\end{tabular}

*According to CT scan and endoscopy. CR: complete remission; PR: partial remission; SD: stable disease; $\mathrm{PD}$ : progression disease; objective response rate $(\mathrm{ORR})=\mathrm{CR}+\mathrm{PR}$; disease control rate $(\mathrm{DCR})=\mathrm{CR}+\mathrm{PR}+\mathrm{SD}$.

and 3 -year overall survival rates are $65 \%$ and $28 \%$, respectively [18]. The main failure pattern of definitive CRT is a locoregional failure. The long-term locoregional control rate is $38 \%-55 \%$, and the predominant site of failure is the primary tumor area [18]. Locoregional failure as the first failure is more common than distant failure $[5,6,9,18,19]$. Locoregional failure is the main failure pattern for ESCC who received definitive radiotherapy. About $40 \%-50 \%$ of patients experience locoregional failure $[6,20]$. In patients who received selective nodal irradiation, primary tumor recurrence is the main failure pattern [21]. Among all the local failures, $90 \%$ of failures are in the GTV, $23 \%$ are in the CTV, and $14 \%$ are in the PTV; $72 \%$ of the patients have failure only in the GTV [9].

Therefore, those data suggest that intensifying local therapy may improve the prognosis of esophageal cancer. Previous studies also demonstrated that a minimum of 65-70 Gy would be needed to achieve tumor control for solid tumors [22]. On the other hand, increasing the radiation dose to esophageal cancer will also increase the dose to the lung, which will increase the risk of severe radiation pneumonitis. Thus, at our center, the target volume definition was modified so that the dose to the tumor could be increased while protecting the lungs at the same time.

Pathological analysis showed that in ESCC, the mean microscopic spread longitudinally beyond the gross tumor is about $10 \mathrm{~mm}$, and $94 \%-97 \%$ of the cases are within $30 \mathrm{~mm}$ [23]. Therefore, the CTV1 was defined as $3 \mathrm{~cm}$ above and below the edge of the GTVt. Both this area and the primary tumor site have a high risk of relapse because the traditional dose of primary tumor and CTV1 is about $50 \mathrm{~Gy}$, which is not a definitive dose. Thus, the dose to the primary tumor and CTV1 was increased to achieve better locoregional control. In the INT0123 trial, the locoregional control was not improved though the dose of GTV was increased to $64.8 \mathrm{~Gy}$, with $1.8 \mathrm{~Gy} /$ day [6]. The INTG0123 trial was carried out using two-dimensional radiotherapy (2DRT), while three-dimensional and IMRT are widely used nowadays. In the present study, the IMRT technique was used, and the dose was increased to $66-68 \mathrm{~Gy}$, with fraction of 2.0$2.2 \mathrm{~Gy} /$ day. The biologically effective dose (BED) was about 72-73 Gy, which is a definitive dose for solid tumors [22]. Because of the high dose in the GTV and CTV1, the V20 of the lungs would be very high if the CTV1 was expanded by $0.5-1.5 \mathrm{~cm}$ around the esophageal wall. Thus, the CTV1 expansion was limited to $1-2 \mathrm{~mm}$ around the esophageal wall.

In terms of toxicity, hematological toxicity was the most common toxicity and was due to chemotherapy. Regarding radiation toxicity, only one patient developed grade 3-4 radiation pneumonitis, and one patient developed grade 3-4 radiation esophagitis. No patients died of toxicity. In the RTOG 8501 trial, the patients who received chemoradiotherapy had more adverse effects than those who received radiotherapy alone, and $10 \%$ had life-threatening toxic effects [5]. The INT0123 trial reported that higher radiotherapy doses were 

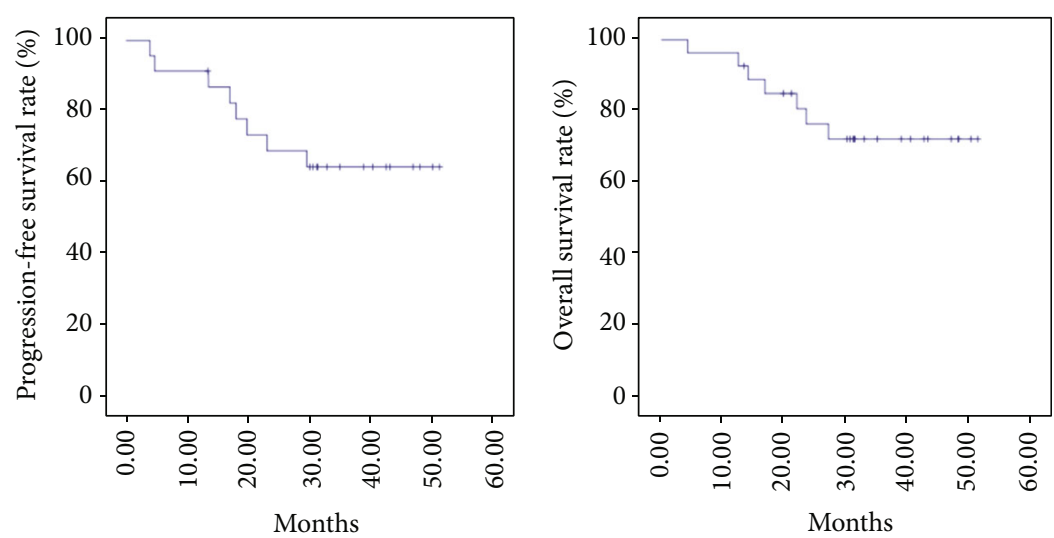

$\neg$ Survival function

+ Censored

(a)

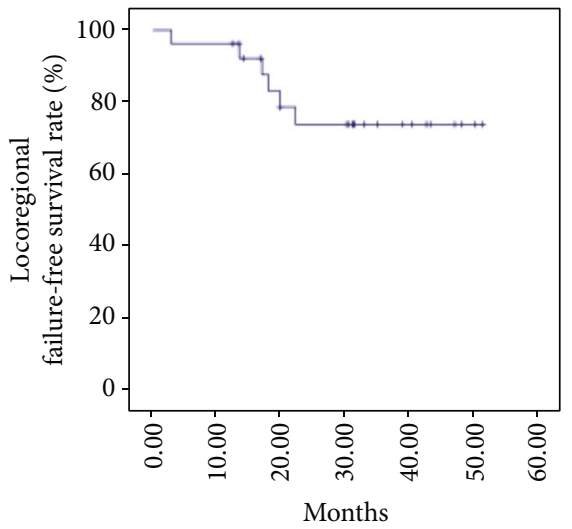

(c) (b)

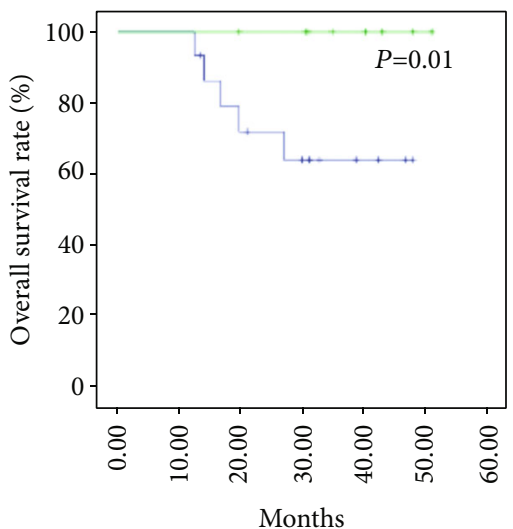

Group

$\neg$ Stage III

$\neg$ Stage IV

+ Stage III censored

+ Stage IV censored

(d)

FIgURe 2: Continued. 


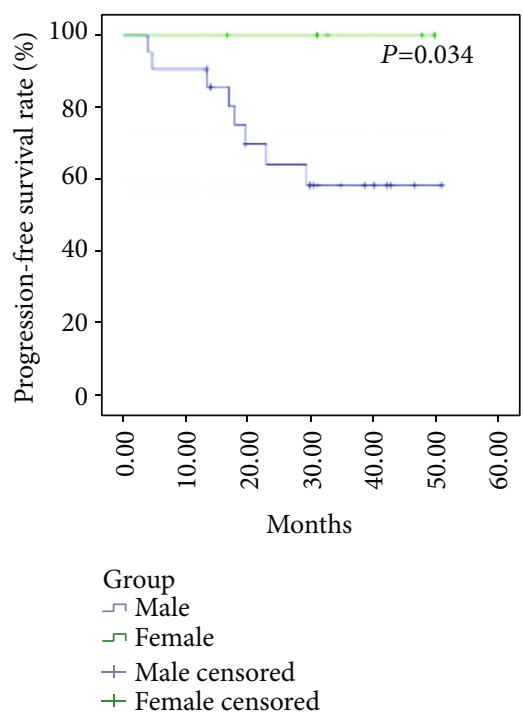

(e)

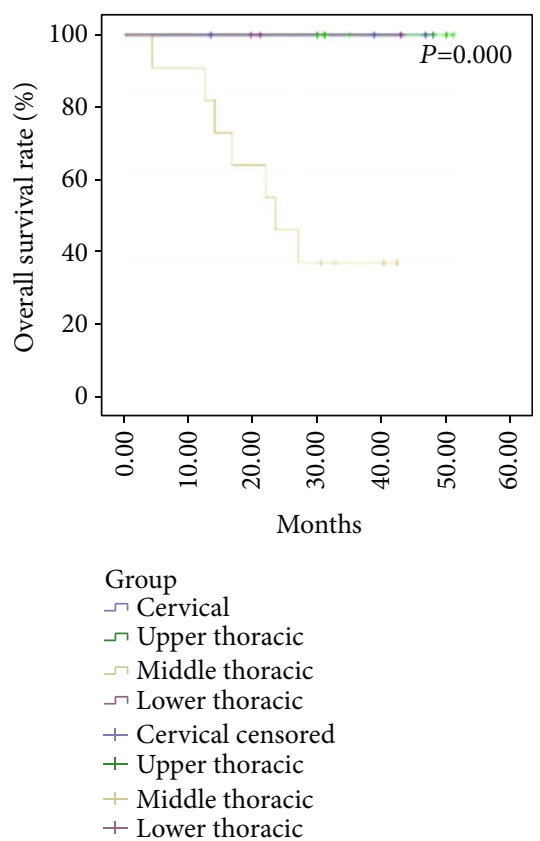

(f)

FIGURE 2: (a-c) Kaplan-Meier survival curves for progression-free survival (PFS), overall survival (OS), and locoregional failure-free survival (LRFFS) curves. (d-f) Subgroup survival curves. (d) The overall survival (OS) of the clinical-stage IV subgroup was longer than that of the stage III subgroup $(P=0.010)$. (e) The progression-free survival (PFS) of female patients was longer than that of males $(P=0.034)$. (f) The OS of the middle-thoracic subgroup was shorter than that of the other subgroups $(P<0.001)$.

TABLE 5: Subgroup analysis for the survival of the patients.

\begin{tabular}{|c|c|c|c|c|c|c|c|c|}
\hline \multirow{2}{*}{ Characteristics } & & \multirow{2}{*}{$N(\%)$} & \multicolumn{2}{|c|}{3 y LRFFS } & \multicolumn{2}{|c|}{3 y PFS } & \multicolumn{2}{|c|}{3 y OS } \\
\hline & & & $\%$ & $P$ & $\%$ & $P$ & $\%$ & $P$ \\
\hline \multirow{2}{*}{ Sex } & Male & $21(80.8)$ & 63.6 & \multirow{2}{*}{0.051} & 57.9 & \multirow{2}{*}{0.034} & 68.4 & \multirow{2}{*}{0.530} \\
\hline & Female & $5(19.2)$ & 100 & & 100 & & 80 & \\
\hline \multirow{2}{*}{ Age (years) } & $\geq 75$ & $3(11.5)$ & 66.7 & \multirow{2}{*}{0.771} & 67.1 & \multirow{2}{*}{0.862} & 76.5 & \multirow{2}{*}{0.069} \\
\hline & $<75$ & $23(88.5)$ & 50.0 & & 50.0 & & 33.3 & \\
\hline \multirow{4}{*}{ Tumor localization } & Cervical & $4(15.4)$ & 66.7 & \multirow{4}{*}{0.059} & 66.7 & \multirow{4}{*}{0.064} & 100 & \multirow{4}{*}{$<0.001$} \\
\hline & Upper thoracic & $8(30.8)$ & 85.7 & & 85.7 & & 100 & \\
\hline & Middle thoracic & $11(42.3)$ & 46.8 & & 46.8 & & 36.4 & \\
\hline & Lower thoracic & $3(11.5)$ & 66.7 & & 66.7 & & 100 & \\
\hline \multirow{2}{*}{$\mathrm{T}$ stage } & 3 & 8 (38.1.) & 85.7 & \multirow{2}{*}{0.202} & 68.6 & \multirow{2}{*}{0.895} & 72.9 & \multirow{2}{*}{0.927} \\
\hline & 4 & $13(61.9)$ & 73.8 & & 73.8 & & 75.5 & \\
\hline \multirow{2}{*}{ Clinical stage } & III & $15(65.2)$ & 60.3 & \multirow{2}{*}{0.067} & 60.3 & \multirow{2}{*}{0.067} & 63.8 & \multirow{2}{*}{0.010} \\
\hline & IV & $8(34.8)$ & 83.3 & & 85.7 & & 100 & \\
\hline \multirow{2}{*}{ Combined with Endostar } & Yes & $14(53.8)$ & 71.4 & \multirow{2}{*}{0.849} & 64.3 & \multirow{2}{*}{0.400} & 78.6 & \multirow{2}{*}{0.201} \\
\hline & No & $12(46.2)$ & 68.8 & & 53.5 & & 61.1 & \\
\hline
\end{tabular}

LRFFS: locoregional failure-free survival; PFS: progression-free survival; OS: overall survival.

related to higher rates of radiation-related deaths [6]. Such toxicity was not observed in the present study, possibly because of the reduced treatment volumes, but this will have to be confirmed in future trials.

Radiation resistance is also an important factor that will impair the effect of radiotherapy. Tumor hypoxia is one of the factors that contribute to radiation resistance [24]. With defects in the endothelium and other structures, blood vessels in tumors are usually structurally and functionally abnormal [25]. These abnormal blood vessels impair the supply of oxygen and results in radiation resistance $[26,27]$. Endostar is a recombinant human endostatin that targets new capillary endothelial cells around tumors [28]. Endostar shows an antitumor effect when it is used together with chemotherapy 
TABLE 6: Treatment-related adverse events.

\begin{tabular}{lccc}
\hline Adverse events, $n(\%)$ & Grade 0 & Grade 1-2 & Grade 3-4 \\
\hline Hematologic toxicity & $3(11.5)$ & $16(61.5)$ & $7(26.9)$ \\
Heart damage & $26(100)$ & 0 & 0 \\
Esophagitis & $10(38.5)$ & $15(57.7)$ & $1(4.5)$ \\
Pneumonitis & $13(50.0)$ & $12(46.2)$ & $1(4.5)$ \\
Skin damage & $26(100)$ & 0 & 0 \\
\hline
\end{tabular}

[28-30]. An in vitro study showed that Endostar inhibits the VEGF signal pathways and thus increases the efficacy of radiotherapy [31]. In the present study, patients treated with Endostar combined with CRT showed a trend towards a survival benefit. Still, no significant difference was observed, possibly because of the small sample size. Future trials should also specifically examine this point.

Middle-thoracic tumor localization was associated with a poor OS. This could be related to Endostar. The numbers of patients with cervical, upper thoracic, middle thoracic, and lower-thoracic tumor localization and who received Endostar are $3(75 \%), 5(62.5 \%), 5(45.5 \%)$, and 1 (33.3\%), respectively. Therefore, the proportion of patients receiving Endostar in the middle-thoracic tumor subgroup was low, contributing to a poorer OS. Although the proportion was low in lowerthoracic tumors as well, there were only three patients in this subgroup, and the sample size was too small to draw any definite conclusion. Furthermore, stage III patients had poorer survival than stage IV patients. The small sample size and the use of Endostar could also explain this paradoxical result. In stage III patients, $7 / 15(46.7 \%)$ received Endostar, while in stage IV patients, 6/8 (75\%) received Endostar. This might contribute to a better OS in stage IV patients. According to the subgroup analysis, patients receiving Endostar tended to have better 3-year LRFFS, PFS, and OS, although no statistically significant difference was observed. Future large sample size studies should verify this analysis.

There are some limitations to this study. First, tumor responses were evaluated by CT scan and endoscopy rather than pathological examination. CT is a poor diagnostic tool to evaluate the tumor response in patients after CRT [32]. Second, the number of patients in this study was not enough to draw any firm conclusions. Third, this is a retrospective analysis, and recall bias may exist. Finally, the observation period is short.

\section{Conclusion}

In conclusion, the modified target volume definition and increased dose of definitive chemoradiotherapy in patients with ESCC had low toxicity and might improve survival, but additional trials are necessary to prove the superiority of this strategy.

\section{Abbreviations}

ESCC: $\quad$ Esophageal squamous cell carcinoma

PGTV: $\quad$ Planning gross tumor volume

PTV1: $\quad$ Planning clinical tumor volume
PFS: $\quad$ Progression-free survival

OS: $\quad$ Overall survival

LRFFS: $\quad$ Locoregional failure-free survival

IMRT: Intensity-modulated radiation therapy

3DCRT: $\quad$ Three-dimensional radiation

GTV: Gross tumor volume

CTV: Clinical target volume

GTVt: $\quad$ Primary tumor

GTVnd: Involved lymph node

PGTVt: Planning gross target volume

PGTVnd: $\quad$ Planning gross tumor volume of lymph nodes

PTV2: $\quad$ Planning target volumes of CTV2

CR: $\quad$ Complete response

PR: $\quad$ Partial response

SD: $\quad$ Stable disease

PD: $\quad$ Progressive disease

ORR: $\quad$ Objective response rate

DCR: Disease control rate

NCI CTCAE: National Cancer Institute Common Terminology Criteria for Adverse Events

2DRT: Two-dimensional radiotherapy.

\section{Data Availability}

The datasets used and/or analyzed during the current study are available from the corresponding authors on reasonable request.

\section{Ethical Approval}

This study was approved by the Ethics Committee of Sun Yat-sen University Cancer Center.

\section{Consent}

Written informed consent was obtained prior to the study.

\section{Conflicts of Interest}

The authors declare that they have no conflicts of interests.

\section{Authors' Contributions}

XP contributed to the article writing, case data collection, and follow-up. ML participated in the data collection follow-up and article modification. HM did the data collection followup and statistical analysis. XJ contributed to the research design and data statistical analysis. $\mathrm{HH}$ participated in the article writing and statistical analysis of data. MW contributed to the project design and article modification. QL contributed to the project design, guided the project throughout the process, and modified the article. All authors have read and approved the manuscript. Xiaofen Pan, Mingchu Liao, and Hongmei Ma contributed equally to this work. 


\section{Acknowledgments}

We are indebted to all colleagues of the Department of Radiation Oncology of Sun Yat-sen University Cancer Center, who provided their assistance for this study.

\section{References}

[1] F. Bray, J. Ferlay, I. Soerjomataram, R. L. Siegel, L. A. Torre, and A. Jemal, "Global cancer statistics 2018: GLOBOCAN estimates of incidence and mortality worldwide for 36 cancers in 185 countries," CA: A Cancer Journal for Clinicians, vol. 68, no. 6, pp. 394-424, 2018.

[2] S. Jain and S. Dhingra, "Pathology of esophageal cancer and Barrett's esophagus," Annals of Cardiothoracic Surgery, vol. 6, no. 2, pp. 99-109, 2017.

[3] Y. Song, L. Li, Y. Ou et al., "Identification of genomic alterations in oesophageal squamous cell cancer," Nature, vol. 509, no. 7498, pp. 91-95, 2014.

[4] D. P. Kelsen, K. A. Winter, L. L. Gunderson et al., "Long-term results of RTOG trial 8911 (USA Intergroup 113): a random assignment trial comparison of chemotherapy followed by surgery compared with surgery alone for esophageal cancer," Journal of Clinical Oncology, vol. 25, no. 24, pp. 3719-3725, 2007.

[5] J. Tepper, M. J. Krasna, D. Niedzwiecki et al., "Phase III trial of trimodality therapy with cisplatin, fluorouracil, radiotherapy, and surgery compared with surgery alone for esophageal cancer: CALGB 9781," Journal of Clinical Oncology, vol. 26, no. 7, pp. 1086-1092, 2008.

[6] B. D. Minsky, T. F. Pajak, R. J. Ginsberg et al., "INT 0123 (Radiation Therapy Oncology Group 94-05) phase III trial of combined-modality therapy for esophageal cancer: high-dose versus standard-dose radiation therapy," Journal of Clinical Oncology, vol. 20, no. 5, pp. 1167-1174, 2002.

[7] C. L. Chang, H. C. Tsai, W. C. Lin et al., "Dose escalation intensity-modulated radiotherapy-based concurrent chemoradiotherapy is effective for advanced-stage thoracic esophageal squamous cell carcinoma," Radiotherapy and Oncology, vol. 125, no. 1, pp. 73-79, 2017.

[8] D. Du, T. Song, X. Liang, M. Fang, and S. Wu, "Concurrent chemoradiotherapy with elective lymph node irradiation for esophageal cancer: a systemic review and pooled analysis of the literature," Diseases of the Esophagus, vol. 30, no. 2, pp. 1-9, 2017.

[9] J. Welsh, S. H. Settle, A. Amini et al., "Failure patterns in patients with esophageal cancer treated with definitive chemoradiation," Cancer, vol. 118, no. 10, pp. 2632-2640, 2012.

[10] S. H. Lin, L. Wang, B. Myles et al., "Propensity score-based comparison of long-term outcomes with 3-dimensional conformal radiotherapy vs intensity-modulated radiotherapy for esophageal cancer," International Journal of Radiation Oncology • Biology • Physics, vol. 84, no. 5, pp. 1078-1085, 2012.

[11] K. J. Napier, M. Scheerer, and S. Misra, "Esophageal cancer: a review of epidemiology, pathogenesis, staging workup and treatment modalities," World Journal of Gastrointestinal Oncology, vol. 6, no. 5, pp. 112-120, 2014.

[12] NCCN, CLinical Practice Guidelines in Oncology (NCCN Guidelines). Esophageal and Esophagogastric Junction Cancers. Version 2.2019, National Comprehensive Cancer Network, Fort Washington, 2019.
[13] A. J. Wu, W. R. Bosch, D. T. Chang et al., "Expert consensus contouring guidelines for intensity modulated radiation therapy in esophageal and gastroesophageal junction cancer," International Journal of Radiation Oncology • Biology • Physics, vol. 92, no. 4, pp. 911-920, 2015.

[14] L. H. Schwartz, S. Litière, E. de Vries et al., "RECIST 1.1Update and clarification: From the RECIST committee," European Journal of Cancer, vol. 62, pp. 132-137, 2016.

[15] A. Trotti, A. D. Colevas, A. Setser et al., "CTCAE v3.0: development of a comprehensive grading system for the adverse effects of cancer treatment," Seminars in Radiation Oncology, vol. 13, no. 3, pp. 176-181, 2003.

[16] V. Grégoire, P. Levendag, K. K. Ang et al., "CT-based delineation of lymph node levels and related CTVs in the node- negative neck: DAHANCA, EORTC, GORTEC, NCIC,RTOG consensus guidelines," Radiotherapy and Oncology, vol. 69, no. 3, pp. 227-236, 2003.

[17] V. W. Rusch, H. Asamura, H. Watanabe et al., "The IASLC lung cancer staging project: a proposal for a new international lymph node map in the forthcoming seventh edition of the TNM classification for lung cancer," Journal of Thoracic Oncology, vol. 4, no. 5, pp. 568-577, 2009.

[18] E. Versteijne, H. W. van Laarhoven, J. E. van Hooft et al., "Definitive chemoradiation for patients with inoperable and/or unresectable esophageal cancer: locoregional recurrence pattern," Diseases of the Esophagus, vol. 28, no. 5, pp. 453-459, 2015.

[19] M. Xi, C. Xu, Z. Liao et al., "The impact of histology on recurrence patterns in esophageal cancer treated with definitive chemoradiotherapy," Radiotherapy and Oncology, vol. 124, no. 2, pp. 318-324, 2017.

[20] K. L. Zhao, J. B. Ma, G. Liu, K. L. Wu, X. H. Shi, and G. L. Jiang, "Three-dimensional conformal radiation therapy for esophageal squamous cell carcinoma: is elective nodal irradiation necessary?," International Journal of Radiation Oncology • Biology - Physics, vol. 76, no. 2, pp. 446-451, 2010.

[21] H. Yamashita, K. Okuma, R. Wakui, S. Kobayashi-Shibata, K. Ohtomo, and K. Nakagawa, "Details of recurrence sites after elective nodal irradiation (ENI) using 3D-conformal radiotherapy (3D-CRT) combined with chemotherapy for thoracic esophageal squamous cell carcinoma-a retrospective analysis," Radiotherapy and Oncology, vol. 98, no. 2, pp. 255-260, 2011.

[22] L. J. Shukovsky and G. H. Fletcher, "Time-dose and tumor volume relationships in the irradiation of squamous cell carcinoma of the tonsillar fossa," Radiology, vol. 107, no. 3, pp. 621-626, 1973.

[23] X. S. Gao, X. Qiao, F. Wu et al., "Pathological analysis of clinical target volume margin for radiotherapy in patients with esophageal and gastroesophageal junction carcinoma," International Journal of Radiation Oncology • Biology • Physics, vol. 67, no. 2, pp. 389-396, 2007.

[24] M. B. Meng, X. D. Jiang, L. Deng et al., "Enhanced radioresponse with a novel recombinant human endostatin protein via tumor vasculature remodeling: experimental and clinical evidence," Radiotherapy and Oncology, vol. 106, no. 1, pp. 130-137, 2013.

[25] H. F. Dvorak, "Vascular permeability factor/vascular endothelial growth factor: a critical cytokine in tumor angiogenesis and a potential target for diagnosis and therapy," Journal of Clinical Oncology, vol. 20, no. 21, pp. 4368-4380, 2002.

[26] M. Nordsmark, S. M. Bentzen, V. Rudat et al., "Prognostic value of tumor oxygenation in 397 head and neck tumors after 
primary radiation therapy. An international multi-center study," Radiotherapy and Oncology, vol. 77, no. 1, pp. 18-24, 2005.

[27] M. W. Dewhirst, Y. Cao, and B. Moeller, "Cycling hypoxia and free radicals regulate angiogenesis and radiotherapy response," Nature Reviews Cancer, vol. 8, no. 6, pp. 425-437, 2008.

[28] S. Zhou, L. Zuo, X. He, J. Pi, J. Jin, and Y. Shi, "Efficacy and safety of rh-endostatin (Endostar) combined with pemetrexed/cisplatin followed by rh-endostatin plus pemetrexed maintenance in non-small cell lung cancer: a retrospective comparison with standard chemotherapy," Thoracic Cancer, vol. 9, no. 11, pp. 1354-1360, 2018.

[29] J. Chen, Q. Yao, M. Huang et al., “A randomized phase III trial of neoadjuvant recombinant human endostatin, docetaxel and epirubicin as first-line therapy for patients with breast cancer (CBCRT01)," International Journal of Cancer, vol. 142, no. 10, pp. 2130-2138, 2018.

[30] Z. Ma, L. Guo, X. Cui, H. Liu, and L. Liu, "Rh-endostatin concomitant with chemotherapy versus single agent chemotherapy for treating soft tissue and bone sarcomas: a systematic review and meta-analysis," Journal of Pharmacy \& Pharmaceutical Sciences, vol. 21, no. 1, pp. 386-397, 2018.

[31] G. F. Liu, H. Chang, B. T. Li et al., "Effect of recombinant human endostatin onradiotherapy for esophagus cancer," Asian Pacific Journal of Tropical Medicine, vol. 9, no. 1, pp. 86-90, 2016.

[32] D. R. Jones, L. A. Parker Jr., F. C. Detterbeck, and T. M. Egan, "Inadequacy of computed tomography in assessing patients with esophageal carcinoma after induction chemoradiotherapy," Cancer, vol. 85, no. 5, pp. 1026-1032, 1999. 